\title{
ABORDAGENS PEDAGÓGICAS NA LITERATURA SOBRE A APRENDIZAGEM MÓVEL NO ENSINO FUNDAMENTAL
}

\author{
K. A. S. NASCIMENTO ${ }^{1}$, J. A. CASTRO FILHO ${ }^{1}$ \\ ${ }^{1}$ Universidade Federal do Ceará \\ karla@virtual.ufc.br
}

Submetido 14/07/2015 - Aceito 04/09/2017

DOI: $10.15628 /$ holos.2017.3216

\section{RESUMO}

Neste trabalho são apresentados os resultados obtidos de uma revisão sistemática da literatura sobre aprendizagem móvel no ensino fundamental. Tem como objetivo apresentar evidências empíricas na utilização de dispositivos móveis que auxiliam o processo de ensino e aprendizagem de professores e alunos do 5으 ao 9o ano. Desta forma, buscou-se analisar pesquisas que tratam sobre as estratégias pedagógicas do uso de dispositivos móveis na escola, como também em aulas de campo. As pesquisas relacionadas ao objetivo central deste estudo admitiram o levantamento científico nas
\end{abstract}

seguintes fontes: banco de teses e periódicos da Coordenação de Aperfeiçoamento de Pessoal de Nível Superior (CAPES), além dos mais conhecidos indexadores de literatura científica de abrangência temática geral: Scientific Electronic Library Online SciELO e Web of Science com as listas de referências citadas de artigos considerados mais importantes por especialistas em sua área de atuação. Os resultados mostraram uma carência de pesquisas que abordem a aprendizagem móvel e seus aplicativos no Ensino Fundamental.

PALAVRAS-CHAVE: Aprendizagem Móvel, Prática Colaborativa, Dispositivos Móveis, Ensino Fundamental.

\section{EDUCATIONAL APPROACHES IN LITERATURE ON MOBILE LEARNING IN ELEMENTARY SCHOOL}

\begin{abstract}
This paper presents the results of a systematic review of the literature on mobile learning in elementary school. It aims to present empirical evidence on the use of mobile devices that support the teaching and learning process of teachers and students of elementary school 5 th to 9 th grade. Thus, it sought to analyze research that deal with the pedagogical strategies of using mobile devices in school, but also in field classes. The research related to the central objective of this study admitted the
\end{abstract}

scientific survey from the following sources: Bank of theses and journals of Higher Education Personnel Improvement Coordination (CAPES), and the most popular scientific literature indexers overall thematic scope: Scientific Electronic Library online SciELO and Web of Science with lists of references cited articles deemed most important by experts in their area. The results showed lack of research that addresses mobile learning and its applications in elementary school.

KEYWORDS: Mobile Learning, Collaborative Practice, Mobile Devices, Elementary School. 


\section{INTRODUÇÃO}

Dispositivos móveis, como tablets, smartphones e laptops estão se tornando cada vez mais populares em todo o mundo, exercendo uma grande influência na maneira como as pessoas acessam informações e se comunicam. Estas tendências têm motivado pesquisas sobre a importância da aprendizagem móvel, principalmente relacionadas à mobilidade do estudante ao utilizar esses dispositivos a fim de facilitar a aprendizagem em vários contextos, envolvendo diferentes locais, atividades e modos de interação entre seus pares e comunidade em geral. Os avanços dessas tecnologias sem fio resultaram no estudo da aprendizagem móvel, possibilitando diversas aplicações, tais como: prática colaborativa, comunicação síncrona e assíncrona, compartilhamento de dados, produção colaborativa, leitor de música e gravação de áudio e vídeo.

A aprendizagem móvel, conforme Sharples et al (2010), pode ser amplamente definida como a exploração de tecnologias móveis onipresentes em conjunto com as redes de telefonia sem fio e móvel, para facilitar, apoiar, melhorar e ampliar o alcance do ensino e da aprendizagem. Esta pode ser trabalhada em qualquer espaço e tempo, visto que envolve conectividade por meio de redes sem fio ou redes de telefonia móvel.

Uma série de publicações tem proporcionado reflexões e discussões sobre a utilização desses dispositivos móveis na educação (BARBOUR, et al., 2014; ROSCHELLE, et al., 2010; SHARPLES, et al. 2010; TING, 2013; TRAXLER, 2009). As iniciativas de investigação têm verificado o seu potencial no processo educativo e, em particular, os benefícios de incorporá-los em sala de aula para desencadear práticas colaborativas e participação ativa do aluno. Para Sharples et al (2010) esta crescente disponibilidade de recursos educacionais abertos está tornando o acesso às informações de forma dinâmica para quem quer aprender. Salientam ainda que se deve aproveitar essa diversidade de tecnologias móveis para oferecer educação aos estudantes em qualquer lugar e a qualquer hora.

Embora não exista uma definição específica para aprendizagem móvel, é importante entender que a mobilidade não é somente o movimento espacial, mas também as maneiras em que tal movimento pode permitir mudanças no tempo e demandar ações que minimizem fronteiras (TRAXLER, 2009).

Em relação à colaboração, conforme Damiani (2008, p. 3), "os membros de um grupo se apoiam, visando atingir objetivos comuns negociados pelo coletivo, estabelecendo relações que tendem a não-hierarquização, liderança compartilhada, confiança mútua e co-responsabilidade pela condução das ações". Em consonância, Torres e Irala (2014, p. 61) destacam que "o conhecimento é construído socialmente, na interação entre pessoas". Os autores exemplificam que quando os alunos são envolvidos em situações-problema, nas quais devem tomar decisões, formando juízos de valor e avaliando implicações sociais, a aprendizagem se torna mais contextualizada.

Apesar disso, ainda há relativamente pouco entendimento acerca das formas como as tecnologias móveis podem ser projetadas e usadas para dar suporte às práticas colaborativas na escola. Zuritta e Nussbaum (2009) sugerem que a mobilidade destes dispositivos possibilita colaboração entre alunos, a fim de estabelecer o movimento e interação com outros em 
ambientes diferentes, ao invés de limitar a atividade com um parceiro alocado em um computador de mesa dentro de um laboratório.

No entanto, o ensino e a aprendizagem apoiados em tecnologias móveis colocam desafios para pesquisadores, profissionais de educação e programadores na criação de ferramentas educacionais para fins didáticos claramente definidos que respondam às reais necessidades dos professores e alunos. Dessa forma, as experiências de aprendizagem que atravessam fronteiras espaciais, temporais e envolvem interações com tecnologias móveis, levaram professores e pesquisadores a refletirem sobre estratégias pedagógicas na utilização destes dispositivos para promover o ensino e a aprendizagem, por sua vez retratadas na literatura.

Nesse contexto, há uma nítida necessidade de se analisar a produção científica nacional e internacional sobre o processo de aprendizagem móvel e a utilização de dispositivos móveis no Ensino Fundamental a partir de uma revisão sistemática da literatura. Segundo Keele (2007), esta revisão pode ser realizada por meio de dois elementos fundamentais: apontar lacunas existentes em uma determinada área de pesquisa ou prover uma coleção proeminente de trabalhos relacionados que podem apoiar novas pesquisas.

Neste sentido, a revisão sistemática aqui realizada tem como questão principal: Qual o panorama atual das publicações científicas nacionais e internacionais sobre a aprendizagem móvel na prática colaborativa e a utilização de dispositivos móveis no Ensino Fundamental? Para respondê-la, foram definidos e analisados dois aspectos: 1) as metodologias e resultados abordados nos estudos sobre aprendizagem móvel na prática colaborativa; 2) os tipos de dispositivos móveis usados no ensino fundamental.

Portanto, o objetivo deste trabalho é apresentar evidências empíricas na utilização de dispositivos móveis (laptop, ipod, iphone, tablet e smartphone) que auxiliam o processo de ensino e aprendizagem de professores e alunos do Ensino Fundamental. Desta forma, buscou-se analisar pesquisas que tratam sobre as estratégias pedagógicas do uso desses dispositivos na escola, como também em aulas de campo.

\section{REVISÃO SISTEMÁTICA DA LITERATURA}

A revisão sistemática é um tipo de investigação científica baseada em evidências, cujos resultados de pesquisas são coletados, categorizados, avaliados e sintetizados. Em 1995, um grupo de cientistas alemães "definiu como revisão sistemática a aplicação de estratégias científicas que limitem o viés de seleção de artigos, avaliem com espírito crítico os artigos e sintetizem todos os estudos relevantes em um tópico específico" (KEELE, 2007, p. 12).

Essa revisão é uma síntese rigorosa de todas as pesquisas relacionadas em uma questão particular. Ela é diferente da tradicional, já que ultrapassa possíveis vieses em todas as fases, seguindo uma metodologia rígida de busca e seleção de pesquisas; analisa a legitimidade do que foi encontrado; coleta, sintetiza e interpreta os dados provenientes das investigações (ROTHER, 2007).

Segundo Barros et al. (2008) a revisão sistemática tem as seguintes etapas:

1. selecionar todos os artigos científicos relevantes, relacionados à questão específica; 
2. fazer a leitura dos resumos das publicações coletadas e eleger aquelas que satisfazem critérios determinados;

3. citar os resultados dos artigos selecionados na etapa anterior por meio de tabelas;

4. de modo opcional, unificar os resultados individuais de cada publicação utilizando técnicas estatísticas.

Frente ao exposto e buscando apresentar elementos que favoreçam reflexões para a aplicação de revisões sistemáticas no cenário da aprendizagem móvel na prática colaborativa e a utilização de dispositivos móveis no Ensino Fundamental, os próximos itens têm como objetivo apresentar as fases que compuseram a revisão sistemática deste estudo e os seus resultados.

\section{PROCEDIMENTOS METODOLÓGICOS}

A fim de selecionar pesquisas relacionadas ao escopo central deste estudo, admitiu-se o levantamento científico nas seguintes fontes: banco de teses e periódicos da Coordenação de Aperfeiçoamento de Pessoal de Nível Superior (CAPES); além dos mais conhecidos indexadores de literatura científica de abrangência temática geral, Scientific Electronic Library Online - SciELO (nacional e internacional) ${ }^{1}$ e Web of Science ${ }^{2}$ (internacional) com as listas de referências citadas de artigos considerados mais importantes por especialistas em sua área de atuação. Destas fontes, foi possível identificar indexadores específicos, tais como: Education Resources Information Center (ERIC) e Science Direct, bibliotecas online digitais e contêm pesquisas na área de Educação e temas relacionados, incluindo mais de 3.000 (três mil) títulos, com disponibilidade de acesso desde 1966 até o presente ano.

Para os critérios de busca foram definidas palavras-chave para identificar estudos sobre aprendizagem móvel na prática colaborativa. Assim, buscou-se as expressões: aprendizagem móvel e prática colaborativa, revisadas por pares com ajuda dos filtros para acompanhar, analisar e visualizar os estudos. O mesmo procedimento foi feito para os termos: dispositivos móveis, tecnologias móveis e ensino fundamental. Estas palavras também foram traduzidas para o inglês e, consequentemente, agregadas à pesquisa.

Todas as ferramentas de buscas utilizadas possuem filtros que delimitam o assunto que se deseja avaliar: umas utilizam "and", "e", "ou", "or", outras possuem campos de data de publicação, tipo de material, idioma. Deste modo, foi realizada, em duas etapas, uma filtragem para a seleção dos artigos, dissertações e teses nacionais e internacionais com o objetivo de coletar maiores informações.

$\mathrm{Na}$ primeira etapa foi feita uma pré-seleção dos periódicos pertinentes à questão principal, lendo os seus títulos, resumos e palavras-chave. Na segunda, todos os pré-selecionados foram avaliados pela pesquisadora e a cada um deles foram identificadas como as palavras-chave foram reveladas, aplicadas e trabalhadas. Em seguida, os dados dos estudos proveram respostas à pergunta oriunda desta revisão sistemática. As pesquisas que estavam em mais de uma base de

\footnotetext{
${ }^{1}$ Disponível em http://www.scielo.org/php/index.php

2 Disponível em

http://apps.webofknowledge.com/UA_GeneralSearch_input.do?product=UA\&SID=4Cb41T93mRyg1K3pZ5B\&search_mode=GeneralSearch
} 
dados diferente foram removidas para evitar duplicações. Por último, os dados dos artigos coletados foram sintetizados para apresentação dos resultados.

\section{RESULTADOS E DISCUSSÕES}

A busca resultou em 2 (duas) dissertações de mestrado e 108 (cento e oito) artigos, publicados em revistas científicas: 20 (vinte) nacionais e 88 (oitenta e oito) internacionais, totalizando 110 (cento e dez) estudos. Apesar de adicionar o termo "ensino fundamental" à pesquisa a fim de especificar melhor a pesquisa, todas as quatro ferramentas de busca compilaram também outros níveis e modalidades de ensino. De tal modo, verificou-se que 98 (noventa e oito) foram eliminados, porque se referiam ao Ensino Superior ou à Educação a Distância ou Educação Profissional ou não apresentavam uma pesquisa empírica sobre a utilização dos dispositivos móveis em práticas colaborativas, apresentado no gráfico seguinte.

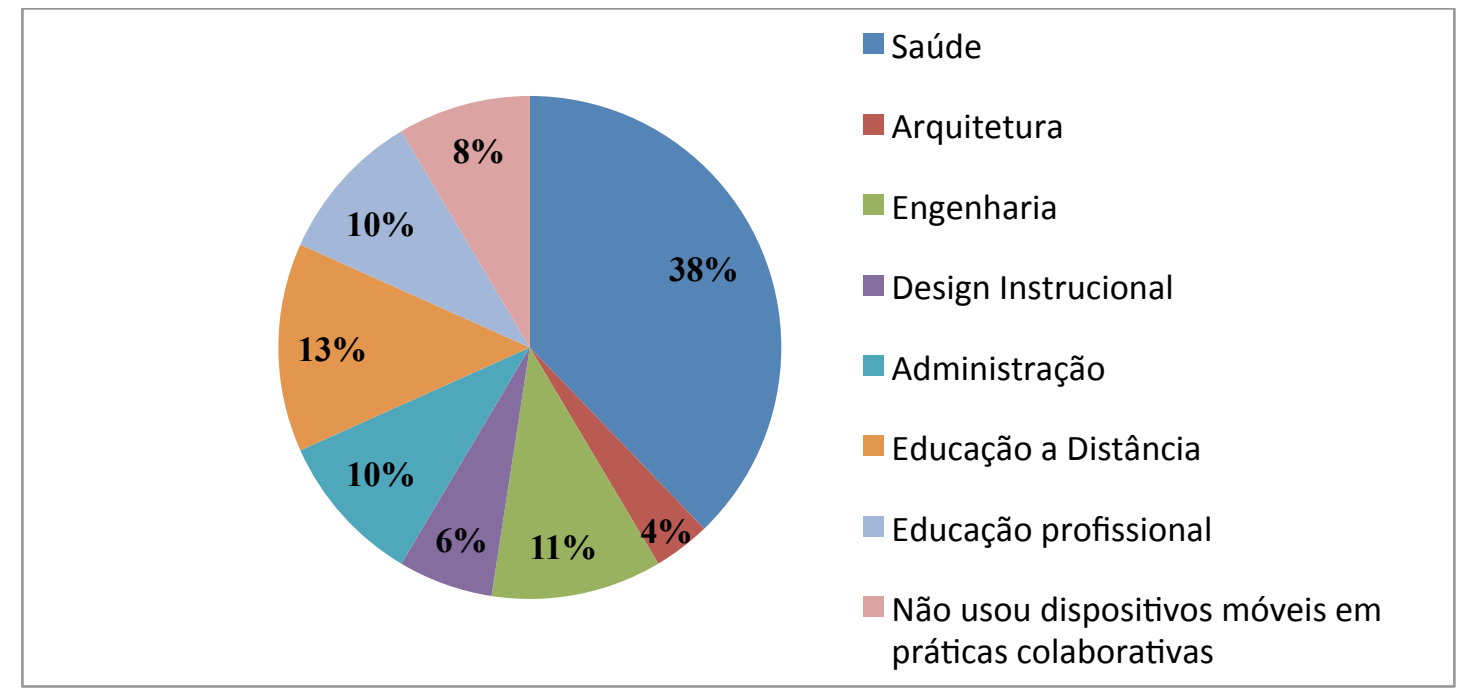

Gráfico 1: Áreas de conhecimento encontradas na pesquisa.

Ressalta-se ainda que o termo "ensino fundamental" e sua tradução para o inglês (elementary e middle school) foi essencial na busca, pois apresenta um público específico de estudante, conteúdo e nível escolar para responder ao objetivo principal deste estudo que pretende apresentar um panorama atual das publicações científicas nacionais e internacionais sobre a aprendizagem móvel na prática colaborativa e a utilização de dispositivos móveis. Desta forma, na segunda triagem, com base no critério de escolha relacionado ao Ensino Fundamental, foi possível extrair e analisar somente 12 (doze) estudos que se detinham ao público-alvo de estudantes do 4ㅇ ao 9ano. A Tabela 1 expõe o total de resultados encontrados em cada base de dados, bem como apresenta o quantitativo dos dados que são relevantes ao problema e às palavras-chave citadas no início deste texto.

Tabela 1: Quantitativo dos resultados nacionais e internacionais.

\begin{tabular}{l|c|c|c|c}
\hline \multirow{2}{*}{ Base de dados } & \multicolumn{2}{|c|}{ Resultados encontrados } & \multicolumn{2}{c}{ Resultados relevantes } \\
\cline { 2 - 5 } & Nacionais & Internacionais & Nacionais & Internacionais \\
\hline Periódicos CAPES & 14 & 23 & 0 & 7 \\
\hline ERIC & 0 & 4 & 0 & 2 \\
\hline
\end{tabular}




\begin{tabular}{l|c|c|c|c}
\hline SciELO & 6 & 7 & 0 & 0 \\
\hline Science Direct & 0 & 54 & 0 & 1 \\
\hline $\begin{array}{l}\text { Banco de teses e } \\
\text { dissertações } \\
\text { CAPES }\end{array}$ & 2 & 0 & 2 & 0 \\
\hline Total & 22 & 88 & 2 & 10 \\
\hline Total Geral & 110 & & 12 \\
\hline
\end{tabular}

Após a leitura dos 12 (doze) estudos relevantes para esta pesquisa, percebeu-se que seus autores usaram, essencialmente, referências de estudos datados entre os anos de 2003 a 2009 com a finalidade de conceituar os termos aprendizagem móvel, prática colaborativa, dispositivos móveis e justificar uma teoria. No entanto, a análise dos textos selecionados está compreendida entre os anos de 2010 a 2015, exposto no gráfico a seguir.

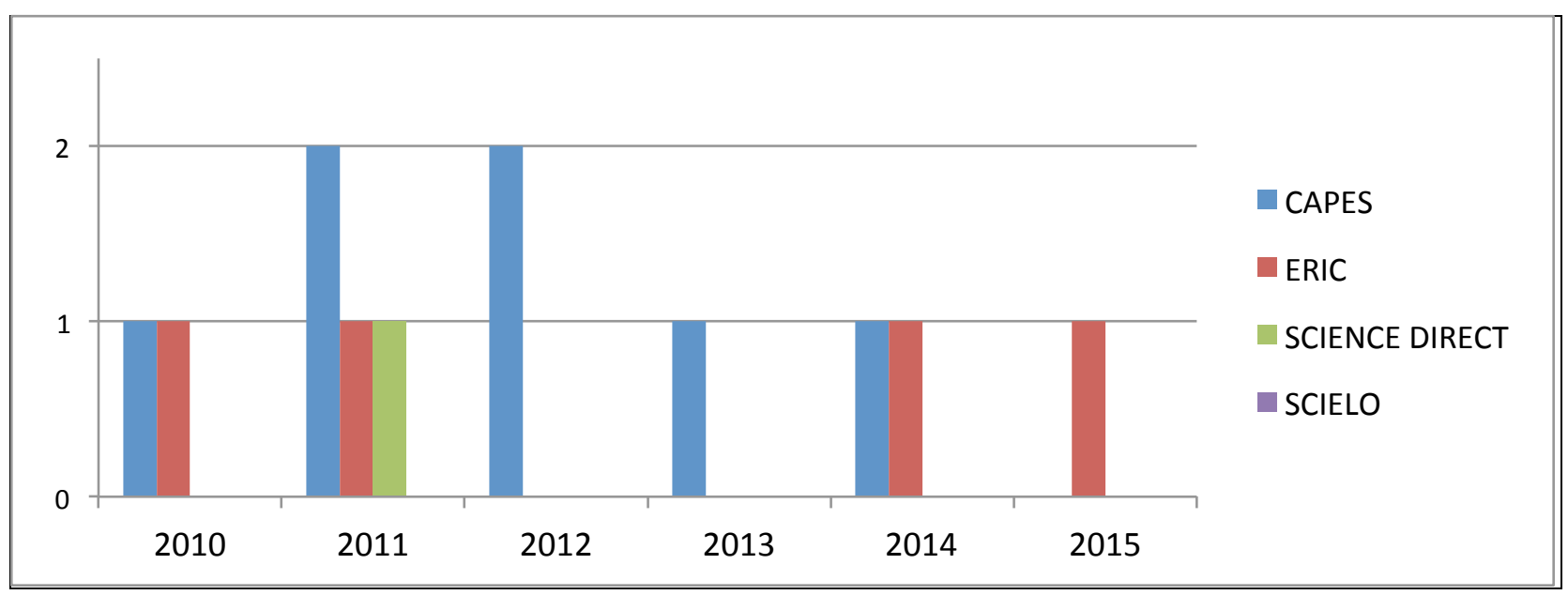

Gráfico 2: Quantidade de artigos por ano.

Em relação aos tipos de aplicativos móveis utilizados, percebe-se que boa parte são recursos fechados (Studywiz, FAO, Mobli21, MLE, TechPals), geralmente elaborados por grupos de pesquisa que ainda estão em processo de desenvolvimento. Outros são livres, mas requerem a criação de um cadastro para ser utilizado (Google Maps, Twitter, Flyer, Wikipédia). Alguns recursos já vêm instalados nos próprios dispositivos móveis, tais como: GPS, áudio book, webcam.

Tabela 2 - Recursos utilizados nos estudos

\begin{tabular}{l|l|l|l|}
\hline Funcionalidade & $\begin{array}{c}\text { Dispositivo } \\
\text { móvel }\end{array}$ & $\begin{array}{c}\text { Recurso/ } \\
\text { Aplicativ } \\
\text { o }\end{array}$ & Atividade \\
\hline $\begin{array}{l}\text { Produção de } \\
\text { textos }\end{array}$ & Smartphone & Studywiz & $\begin{array}{l}\text { Produzir texto colaborativo com ajuda da } \\
\text { plataforma de aprendizagem Studywiz. }\end{array}$ \\
\hline $\begin{array}{l}\text { Representações } \\
\text { gráficas de } \\
\text { frações }\end{array}$ & Palm Tops & FAO & $\begin{array}{l}\text { ldentificar como se dá o apoio das } \\
\text { tecnologias móveis durante o } \\
\text { desenvolvimento da atividade sobre frações. }\end{array}$ \\
\hline Uso da Internet e & Ipod Touch & Áudio & Apoiar a aprendizagem do conteúdo de \\
\hline
\end{tabular}




\begin{tabular}{l|l|l|l}
\hline $\begin{array}{l}\text { o ensino de } \\
\text { língua inglesa }\end{array}$ & $\begin{array}{l}\text { book } \\
\text { Google }\end{array}$ & $\begin{array}{l}\text { línguas, ampliando o tempo de pesquisa na } \\
\text { Internet com atividades realizadas em casa. }\end{array}$ \\
\hline $\begin{array}{l}\text { Compartilhamen } \\
\text { to de imagens }\end{array}$ & Iphone & Flyer & $\begin{array}{l}\text { Solucionar os problemas expostos pelo } \\
\text { professor por meio de uma série de imagens } \\
\text { em sequência (storyboard). }\end{array}$ \\
\hline $\begin{array}{l}\text { Registro das } \\
\text { localizações } \\
\text { espaciais }\end{array}$ & Iphone & $\begin{array}{l}\text { Webcam } \\
\text { Google } \\
\text { Maps, } \\
\text { GPS }\end{array}$ & $\begin{array}{l}\text { Promover a familiaridade dos alunos com o } \\
\text { urbanismo e as características sócio- } \\
\text { geográficas dos diferentes distritos da cidade } \\
\text { de Duc de Montblanc de Rubí na Espanha. }\end{array}$ \\
\hline $\begin{array}{l}\text { Comunicação } \\
\text { online }\end{array}$ & Tablet & Twitter & $\begin{array}{l}\text { Explorar as habilidades de interpretação dos } \\
\text { alunos no Museu, bem como a compreensão } \\
\text { sobre "Igualdade e crenças: os direitos civis". }\end{array}$ \\
\hline $\begin{array}{l}\text { Produção de } \\
\text { textos }\end{array}$ & Tablet & $\begin{array}{l}\text { Wikipédi } \\
\text { a }\end{array}$ & $\begin{array}{l}\text { Produzir textos sobre os espaços da escola } \\
\text { onde cada grupo deverá intervir na produção } \\
\text { do outro. }\end{array}$ \\
\hline $\begin{array}{l}\text { Perguntas e } \\
\text { respostas sobre } \\
\text { História europeia }\end{array}$ & Blackberry & Mobl21 & $\begin{array}{l}\text { Projetar conteúdos de um curso em um } \\
\text { aplicativo móvel e examinar as percepções } \\
\text { dos alunos em relação à aprendizagem. }\end{array}$ \\
\hline $\begin{array}{l}\text { Atividades de } \\
\text { produção }\end{array}$ & Smartphone & MLE & $\begin{array}{l}\text { Desenvolver e organizar as atividades de } \\
\text { produção realizadas na sala de aula em } \\
\text { vários formatos. }\end{array}$ \\
\hline $\begin{array}{l}\text { Ensino de } \\
\text { frações }\end{array}$ & Palm Tops & TechPals & $\begin{array}{l}\text { Solucionar os desafios mediados pelo } \\
\text { docente, ajudando uns aos outros a } \\
\text { aprender sobre frações. }\end{array}$ \\
\hline
\end{tabular}

No Portal de Periódicos CAPES (http://www.periodicos.capes.gov.br/), 7 (sete) artigos tratam sobre os elementos pontuados neste estudo com alguma importância. Destes, 2 (dois) discutem e analisam experiências de utilização de telefones celulares na escola. No primeiro, Norris e Soloway (2010) admitem que os alunos de uma escola localizada na zona rural de Ohio nos EUA, em um projeto realizado em 2008 e 2009, usaram celulares para desenvolver 50\% das suas atividades diárias na escola e, em seguida, usaram-nos para trabalhos fora do ambiente escolar. Os alunos utilizaram uma plataforma de aprendizagem chamada Studywiz que fornece alguns recursos digitais, entre eles um editor de texto colaborativo. Os resultados evidenciam que os estudantes do Ensino Fundamental mostraram melhores desempenhos na escrita. Eles elaboraram textos de forma coletiva que incialmente eram divididos em equipes e depois organizados com todos os alunos da turma.

Em uma atividade colaborativa de matemática, um professor reforçou o currículo, ajustou seus horários de aula e procurou estratégias para identificar pares com frações complementares combinando setores circulares em uma turma de alunos do 4ㅇ ano do Ensino Fundamental de uma escola em Cingapura na China, em seguida, formaram grupos entre eles. Para isso, utilizaram o aplicativo Form-A-One (FAO) que trata as frações na forma de representações gráficas. Boticki et al. (2011) investigaram e mapearam a colaboração de cada grupo com base em três aspectos: tecnológico, social e docente, a fim de identificar como se dá o apoio das tecnologias móveis durante o desenvolvimento da atividade com frações. Segundo os autores, os 
suportes tecnológico e social eram recíprocos, os estudantes se comunicavam e negociavam verbalmente, como também por meio de smarthphones. Além disso, essas tecnologias facilitaram a compreensão dos problemas mediados pelo professor e promoveram avanços nas resoluções dos problemas, pois os alunos visualizavam as frações dos outros, podendo se referir a seus dispositivos e percorrer as listas correspondentes, ajudando, explicando e justificando suas hipóteses. O professor foi capaz de especificar parâmetros na resolução dos problemas que impactaram diretamente na realização e nas possibilidades de colaboração dos alunos, ou seja, em cada etapa, o professor questionava, orientava os alunos em relação aos novos problemas, incentivava-os a percorrer a sala e a negociar.

Em outro estudo, Liu et al. (2014) investigaram uma iniciativa de aprendizagem móvel em um grande distrito escolar nos Estados Unidos a partir do fornecimento de dispositivos de iPod touch $^{3}$ durante todos os dias da semana em qualquer tempo e espaço para professores e alunos de língua inglesa. Os professores apresentavam textos e músicas em inglês e os alunos agregavam outras informações como vídeos e imagens ao banco de tarefas disponibilizado em uma pasta virtual compartilhada. Os resultados revelaram que o iPod touch foi usado para apoiar a aprendizagem do conteúdo de línguas, fornecer apoio pedagógico diferenciado e ampliar o tempo de pesquisa com atividades realizadas em casa. No entanto, alguns desafios foram identificados, tais como: necessidade de formação profissional e pessoal de suporte técnico.

As aulas de campo com a utilização de dispositivos móveis foram destaques em três estudos discutidos a seguir. Laru et al. (2012) identificaram e compararam o desempenho de 22 (vinte e dois) alunos do 7음 ano do Ensino Fundamental, divididos em duas duplas e seis trios, durante discussões argumentativas com apoio de telefone celular, na situação 1:1 (um dispositivo por aluno), realizadas em uma viagem ao parque natural no norte da Finlândia. $O$ projeto consistia em solucionar os problemas expostos pelo professor por meio de uma série de imagens em sequência (storyboard) do aplicativo Flyer que permite criar uma rede social transmitindo e buscando informações de pessoas ou grupos. No projeto, os alunos apresentavam seus próprios argumentos aos questionamentos do professor na forma de mensagens e comparavam com os demais. Segundo os pesquisadores o uso da ferramenta de mensagens promoveu interações argumentativas durante os questionamentos dos alunos o que possibilitou estabelecer níveis das respostas e da participação e, consequentemente, a criação de uma nova sequência de imagens para produção de um vídeo.

Pérez-Sanagustín et al. (2012) recomendam que as atividades colaborativas com apoio dos dispositivos móveis nas aulas de campo devem considerar quatro fatores: o espaço, o método pedagógico, os participantes e a História. Esses fatores compõem o termo 4SPPIces e estabelecem um quadro que produz um roteiro para facilitar o desenvolvimento dessas atividades. Os pesquisadores testaram o 4SPPIces em uma turma com 34 (trinta e quatro) alunos e dois professores (titular e auxiliar). O roteiro teve o propósito de resolver as limitações de uma atividade, que ocorre todos os anos, de trabalho de campo na disciplina de Geografia para promover a familiaridade dos alunos com o urbanismo e as características sócio-geográficas dos diferentes distritos da cidade de Duc de Montblanc de Rubí na Espanha. Os autores revelaram que o fator espaço provou ser um bom mecanismo para utilizar ferramentas disponíveis

\footnotetext{
${ }^{3}$ IPod Touch é um aparelho de mídia portátil com tela de toque desenhado e vendido pela empresa Apple para ouvir, gravar músicas, assistir vídeos e registrar imagens.
} 
(webcam, GPS, pesquisa de mapas) nas tecnologias móveis, dado a possibilidade do registro das localizações espaciais, onde ocorreram as atividades. Devido à organização do projeto, os docentes se limitavam a observar e tirar dúvidas sobre o conteúdo e sua comunicação com os alunos era de forma presencial, pontual e verbal.

Charitonos et al. (2012) investigaram o uso de redes sociais e tecnologias móveis em uma excursão escolar ao Museu de Londres com 29 (vinte e nove) alunos do 9o ano e o professor de História de uma escola de Milton Keynes, localizada a $72 \mathrm{~km}$ de Londres. 0 trabalho pedagógico em museus está ancorado nas diferentes perspectivas socioculturais de aprendizagem com foco na mediação de artefatos para compreensão do que se vê, lê e ouve. Neste sentido, o projeto teve como objetivo explorar as habilidades de interpretação dos alunos, bem como a compreensão sobre o conhecimento disciplinar, especificamente sobre "Igualdade e crenças: os direitos civis", desenvolvidos ao longo do tempo e materializados de várias formas (visual, escrita e oral) e meios de comunicação (aplicativos online). Os alunos foram divididos em oito grupos para coletar algumas evidências apresentadas em três galerias com o uso de tablet e do Twitter, para inserção de notas, fotos e postagens, a fim de criar quatro questões diferentes sobre o assunto e, posteriormente, na sala de aula, criar uma apresentação. Os autores concluíram que a utilização do Twitter melhorou a compreensão, participação e entusiasmo dos alunos durante a visita ao museu. As interações online ajudaram na negociação e na troca de conhecimento entre os estudantes. Além disso, as tecnologias não interromperam e nem perturbaram a autenticidade do cenário, pelo contrário, elas tiveram um impacto sobre a dinâmica social e pedagógica proposta na visita ao museu, como também, auxiliaram o processo de construção de significados de forma compartilhada. Contudo, o papel do professor na pesquisa ficou restrito em observar os alunos e ajudá-los em questões relacionadas à segurança no local, assim, não foi possível compreender a mediação docente e a ligação entre os conteúdos vistos em sala e na aula de campo.

Esse três estudos apontam a possibilidade de registros das informações coletadas nos ambientes externos à escola com uso de aplicativos e dispositivos móveis. Esses registros foram importantes para o desenvolvimento de atividades de comparação, compartilhamento e produção, além de permitir continuidade dos conteúdos curriculares e promover debates sobre o que foi analisado e revelado.

Finalmente, Ting (2013) realizou um estudo para identificar como os alunos do 60 ano do Ensino Fundamental de uma escola de Taiwan na China interagiam-se ao utilizar tecnologias móveis nas atividades escolares. 57 (cinquenta e sete) alunos divididos em 19 (dezenove) grupos participaram deste experimento. Antes de iniciarem as atividades, eles preencheram um questionário sobre conhecimento prévio no uso de tecnologias móveis. $87 \%$ responderam que conheciam, mas não tinham usado, os outros $13 \%$ afirmaram ter utilizado em algum momento fora da escola. A atividade consistia em produzir textos sobre os espaços da escola e cada grupo deveria intervir na produção do outro. Após a atividade, cada participante escreveu um ensaio sobre a tecnologia móvel para medir seu desempenho de aprendizagem. Os resultados deram ênfase à visão dos alunos sobre a utilização de dispositivos móveis no dia a dia da escola e estes foram unânimes em dizer que estas tecnologias permitem melhor interação entre eles e tornam o aprendizado mais prático e repleto de possibilidades, pois possui diversos aplicativos que ajudam no desenvolvimento da atividade. 
O serviço ERIC (http://eric.ed.gov/) de indexação de publicações na área de educação patrocinado pelo departamento de educação do governo americano indexa revistas internacionais de cunho educativo, anais de conferências, relatórios e produções bibliográficas. Desse acervo foram selecionados 2 (dois) artigos.

O primeiro estudo analisou a utilização de um aplicativo que funciona em dispositivos móveis, chamado Mobl21, por 11 (onze) estudantes com idade entre 10 e 12 anos matriculados em um curso suplementar de História Europeia, inicialmente, realizado em ambiente virtual de aprendizagem, oferecido em uma escola no Centro-Oeste dos EUA. Segundo Barbour et al. (2014), a implantação do Mobl21 no lugar do ambiente virtual do curso, na visão dos alunos, causou mudança pedagógica. Este aplicativo auxilia a aprendizagem de diversos temas educativos através de perguntas, apresentação de vídeos, textos e funciona em três sistemas operacionais: iOS, Android e Blackberry, bem como em uma versão emulada que pode ser executada em qualquer dispositivo móvel. Ao longo de quatro semanas usando Mobl21, os alunos acessaram o material didático e foram desafiados a responder questões sobre o conteúdo. Como resultado, as percepções dos alunos sobre Mobl21 foram justificadas pela usabilidade e mobilidade: 1) facilidade de acesso das ferramentas em qualquer dispositivo; 2 ) regularidade nos estudos, visto que a tecnologia está à mão; e, 3) pode ser utilizado em qualquer espaço e tempo. No entanto, o estudo revelou que os alunos tiveram dificuldades em se adaptar às funcionalidades do Mobl21 em seus próprios celulares e sentiram falta da mediação docente: quando tinham dúvidas sobre os conteúdos não podiam recorrer aos fóruns, pois o aplicativo não possuía tal recurso.

Já no segundo experimento, Norris, Hossain e Soloway (2011) apresentam os resultados de um estudo com estudantes do 4을 ano do Ensino Fundamental de uma escola de Cingapura, na China. Foi implantado um aplicativo chamado de Ambiente de Aprendizagem Móvel (Mobile Learning Environment-MLE) e permitiu que todas as atividades fossem expostas no smartphone. Por exemplo, na atividade sobre plantas, os alunos foram convidados a criar um mapa conceitual, uma animação e uma planilha eletrônica. A produção discente é representada no MLE. Neste projeto, os alunos tiveram acesso ao dispositivo de forma integral, ou seja, o smartphone foi usado em $100 \%$ do tempo de aula para desenvolver cada tarefa, bem como fora da escola. No entanto, o estudo mostra que a colaboração é uma habilidade que os professores de Cingapura estão tentando trabalhar com seus alunos devido à necessidade de repensar o currículo para tirar - máximo proveito dos smartphones, implementar estratégias pedagógicas baseadas em indagações que apoiem as metas curriculares e acompanhar o impacto dessa mudança no desempenho dos alunos. Apesar disso, os autores presumem que a utilização de celulares nas escolas possui custo menor e preveem que dentro de 5 (cinco) anos cada criança nos Estados Unidos vai usar um dispositivo e aplicativos de aprendizagem móvel. A investigação observou que a situação $1: 1$, se não for devidamente planejada, oferece pouca vantagem sobre os usos tradicionais da tecnologia.

Na plataforma online americana Science Direct (http://www.sciencedirect.com/), que permite acesso a artigos em texto completo escritos por autores do cenário científico nas principais áreas do conhecimento, foi selecionada 1 (uma) pesquisa sobre a utilização de uma ferramenta colaborativa móvel no ensino de frações, chamada Tecnologia Mediada de 
Aprendizagem Assistida por Pares (Technology-Mediated Peer-Assisted Learning - TechPALS) ${ }^{4}$, para alunos do 4ㅇan ano do Ensino Fundamental em uma escola de São Francisco, na CalifórniaEUA. Roschelle et al. (2010) investigaram que TechPALS fornece evidências de que, para desenvolver práticas colaborativas eficazes, é necessário ampliar o foco para além das ferramentas. Ao utilizar TechPALS os alunos interagiam para solucionar os desafios, mediados pelo docente, ajudando uns aos outros a aprender sobre frações. Os autores reforçam que as atividades devem ser contextualizadas para integrar as ações de colaboração entre os alunos com o currículo. No entanto, a pesquisa demonstra uma experiência restrita em relação à colaboração entre pares, pois a turma de alunos tinha uma série de problemas comportamentais, como também não possuía o mínimo domínio do conteúdo explorado no TechPALS. As notas de campo evidenciaram mais as dificuldades técnicas do que pedagógicas. Isso contribuiu para melhorar as funcionalidades do recurso em questão.

O resultado proveniente do banco de teses e dissertações da CAPES (http://bancodeteses.capes.gov.br/) que efetua somente busca de cursos brasileiros de pósgraduação, revelou 2 (duas) dissertações de Mestrado Acadêmico defendidas no período de 2011 e 2012 que trataram sobre o estudo da aprendizagem móvel na prática colaborativa com apoio dos dispositivos móveis no Ensino Fundamental. Desse critério, seguem dois estudos.

No primeiro, Barbosa Neto (2012) apresenta uma metodologia para desenvolvimento de jogo educativo digital para dispositivos móveis e sua integração a ambientes virtuais de aprendizagem - AVA. A criação do jogo para a disciplina de Matemática do Ensino Fundamental foi dividida em três fases: desenvolvimento dos aplicativos, integração com AVA e avaliação final. Para se tornar atrativo e motivador, o jogo foi dividido em seis estágios, onde cada estágio depende do outro e possui nível de dificuldade diferente. Os participantes da pesquisa que utilizaram o jogo após sua implantação foram: quatro alunos do 8o ano do Ensino Fundamental de uma escola particular e doze alunos do 1 o ano do Ensino Médio de uma escola pública estadual da cidade de Recife-PE. Os resultados indicaram a facilidade dos alunos ao utilizarem os jogos através de smartphone, estimulando-os a avançar nos estágios através de estratégias individuais na resolução de problemas em qualquer momento. A integração com o AVA disponibilizou retorno sobre o progresso dos alunos em cada estágio, bem como a satisfação de aprender o conteúdo de maneira divertida. Embora os alunos tenham utilizado o jogo através de dispositivos móveis, o estudo não apresenta situações de aprendizagem colaborativa entre os alunos durante a execução do jogo e nem do AVA. Percebe-se, entretanto, que as ações foram individuais e proporcionou somente comunicação entre homem e máquina.

No segundo, Higuchi (2011) investigou como o ambiente escolar incorpora a tecnologia móvel no processo educacional. A pesquisadora realizou uma experiência em uma escola pública com uma professora e seus alunos do 9o ano do Ensino Fundamental e 2ㅇ ano do Ensino Médio de uma escola púbica de São Paulo, que utilizaram o aparelho celular para auxiliar atividades pedagógicas desenvolvidas na disciplina de História. O estudo mostra que, para atrair os alunos nas aulas, a professora pediu que registrassem fotos de possíveis focos do mosquito transmissor da dengue na região onde moram, já que todos estavam empenhados no desenvolvimento das tarefas do projeto "Prevenção à Dengue" realizado no 10 semestre de 2010. Perante a

\footnotetext{
${ }^{4}$ TechPALS foi desenvolvido pelo professor Miguel Nussbaum da Universidad Catolica de Santiago, Chile, como também implementado em escolas da Califórnia nos EUA. Tem como objetivo ensinar frações para estudantes do Ensino Fundamental.
} 
curiosidade dos alunos em pesquisar, surgiu a ideia de utilizar seus próprios celulares para fotografar e criar paródias sobre a temática, a fim de conscientizar a população da necessidade de supervisionar ambientes propícios à fecundação do mosquito. Deste modo, compartilharam informações e organizaram debates sobre o assunto. Os resultados da pesquisa mostram a facilidade dos alunos em acessar recursos digitais online através do celular durante as aulas, como também a necessidade de saber mais sobre o tema, tendo como base a pesquisa na web.

A análise dos trabalhos revela que nenhum dos estudos enfatiza o potencial da aprendizagem móvel na prática colaborativa para o desenvolvimento de atividades que vão além do ambiente escolar propriamente dito. Além disso, os estudos não analisaram os dispositivos móveis a partir do ponto de vista do ensino e do seu papel na aprendizagem. Mais importante ainda, o desenvolvimento e o uso de padrões de tecnologia estão mudando rapidamente, exigindo uma análise das tendências e funcionalidades dos diversos tipos de dispositivos móveis na educação, isso também não foi revelado nas investigações. Apesar disso, essas pesquisas relatam resultados positivos que mostram o envolvimento dos alunos com tecnologias móveis, no entanto, é necessário verificar se o que ocorre durante o processo de aprendizagem móvel está sendo também trabalhado e relacionado ao contexto escolar e o que se vive na comunidade em geral, ultrapassando os muros da escola, ou seja, identificar estratégias de como o professor pode mediar práticas colaborativas com dispositivos móveis no contexto da sala de aula em situações presentes no dia a dia do aluno.

\section{CONSIDERAÇÕES FINAIS}

A busca realizada nesta revisão sistemática da literatura resultou na pré-seleção de 108 (cento e oito) estudos, dos quais somente 13 (treze) foram selecionados para a extração de dados, visto que obedeciam aos critérios relacionados à aprendizagem móvel na prática colaborativa, mais especificamente para o Ensino Fundamental. Desses, 3 (três) são provenientes de dissertações de mestrado. A distribuição temporal mostrou que os artigos foram publicados entre os anos de 2010 e 2015. Já a classificação geográfica das instituições de ensino fundamental apresentadas na investigação mostrou que a maior parte delas está localizada em países estrangeiros.

A discrepância entre a quantidade de artigos publicados nos contextos do Ensino superior, Educação a Distância, Educação Profissional e aqueles que não apresentavam um estudo empírico sobre a utilização dos dispositivos móveis em práticas colaborativas na educação básica, revelou claramente a carência de pesquisas que abordem a aprendizagem móvel, prática colaborativa e o uso de seus dispositivos móveis no Ensino Fundamental.

A facilidade do uso desses dispositivos se torna uma opção atraente para sua integração no dia a dia da sala de aula. A característica mais promissora de contextos de aprendizagem móvel é que se pode, de forma colaborativa, realizar diversas atividades tanto dentro da escola como fora dela. Com base nas leituras, compreendeu-se que os resultados de aprendizagem móvel nas práticas colaborativas dependem da mediação docente ao promover atividades em que grupos de estudantes realmente se envolvam em interações produtivas. Assim, foi possível perceber que as pesquisas trouxeram algumas tentativas de trabalhar a colaboração com o apoio de dispositivos móveis, no entanto ficou demonstrado que essas práticas precisam ser aprendidas a partir de um plano que oriente todos os passos que se pretende desenvolver, caso 
contrário tanto o docente quanto o discente podem se sentir perdidos. Neste sentido, Zurita e Nussbaum (2004) sugerem que, para garantir a prática colaborativa entre alunos, é necessário envolvê-los em interações estruturadas, com base em regras prescritas que estabelecem como devem formar grupos, colaborar e resolver problemas.

A organização apresentada para as situações de pesquisas e os tipos de artefatos propostos sugere que há uma preferência nítida dos pesquisadores por construírem ferramentas que apoiem as tecnologias móveis em diversos contextos de aprendizagem, contudo, percebemse poucos estudos sobre a utilização de dispositivos móveis para alunos do Ensino Fundamental.

\section{REFERÊNCIAS}

Barbosa Neto, J. F. B. (2012). Uma Metodologia de Desenvolvimento de Jogos Educativos em Dispositivos Móveis para Ambientes Virtuais de Ensino. Dissertação de Mestrado Acadêmico em Ciência da Computação - Universidade Federal de Pernambuco, Recife.

Barbour, M.; Grzebyk, T. Q.; EYE, J. (2014). Any Time, Any Place, Any Pace-Really? Examining Mobile Learning in a Virtual School Environment. Turkish Online Journal of Distance Education-TOJDE, v. 15, n. 1, 114-127. http://doi.org/10.17718/tojde.45828.

Barros, A. C.; Wainer, J.; Claudio, K.; Ferreira, L. R. R.; Dwyer, T. (2008). Uso de computadores no Ensino Fundamental e Médio e seus resultados empíricos: uma revisão sistemática da literatura. Revista Brasileira de Informática na Educação. 16(1), 57-68. http://dx.doi.org/no\%20doi.

Boticki, I.; Looi, C. K.; Wong, L. H. (2011). Supporting Mobile Collaborative Activities through Scaffolded Flexible Grouping. Educational Technology \& Society, 190-202.

Higuchi, A. A. da S. (2011). Tecnologias móveis na educação. Dissertação de Mestrado Acadêmico em Educação, Arte e História da Cultura - Universidade Presbiteriana Mackenzie de São Paulo, São Paulo.

Damiani, M. F. (2008). Entendendo o trabalho colaborativo em educação e revelando seus benefícios. Educar em revista, n. 31, 213-230. http://doi.org/10.1590/S010440602008000100013.

Charitonos, K.; Blake, C., Scanlon, E.; Jones, A. (2012). Museum learning via social and mobile technologies: (How) can online interactions enhance the visitor experience? British Journal of Educational Technology, v. 43, n. 5, 802-819. http://doi.org/10.1111/j.14678535.2012.01360.x.

Laru, Jari; Järvelä, Sanna; Clariana, R. B. (2012). Supporting collaborative inquiry during a biology field trip with mobile peer-to-peer tools for learning: a case study with $\mathrm{K}-12$ learners. Interactive Learning Environments, v. 20, n. 2, 103-117. http://dx.doi.org/10.1080/10494821003771350.

Liu, M.; Navarrete, C. C.; Wivagg, J. (2011). Potentials of Mobile Technology for K-12 Education: An Investigation of iPod touch Use for English Language Learners in the United States. Educational Technology \& Society, 115-126.

Keele, S. (2007). Guidelines for performing systematic literature reviews in software engineering. Technical report, EBSE Technical Report EBSE. 
Pérez-Sanagustín, M.; Santos, P.; Hernández-Leo, D.; Blat, J. (2012). 4SPPIces: A case study of factors in a scripted collaborative-learning blended course across spatial locations. International Journal of Computer-Supported Collaborative Learning, v. 7, n. 3, 443-465.

Rother, E. T. (2007). Revisão sistemática versus revisão narrativa. Acta Paulista de Enfermagem, v. 20 , n. 2, p. V-VI.

Roschelle, J.; Rafanan, K.; Estrella, G.; Nussbaum, M.; Claro, S. (2010). From handheld collaborative tool to effective classroom module: Embedding CSCL in a broader design framework. Computers \& Education, v. 55, n. 3, 1018-1026. http://doi.org/ 10.1016/j.compedu.2010.04.012.

Sharples, M., Arnedillo-Sánchez, I., Milrad, M.; Vavoula, G. (2010). Mobile learning. Springer Netherlands, p. 233-249.

Norris, C.; Hossain, A.; Soloway, E. (2011). Using Smartphones as Essential Tools for Learning. Educational Technology, v. 51, n. 3, 18-25.

Norris, C. A.; Soloway, E. (2010). Innovative Leaders Take the Phone and Run: Profiles of Four Trailblazing Programs. District Administration, v. 46, n. 6, 35.

Ting, Y. (2013). Using mobile technologies to create interwoven learning interactions: An intuitive design and its evaluation. Computers \& Education, v. 60, n. 1, 1-13. DOI: 10.1016/j.compedu.2012.07.004.

Torres, P. L.; Irala, E. A. F. (2014). Aprendizagem colaborativa: teoria e prática. Complexidade: redes e conexões na produção do conhecimento. Curitiba: Senar, p. 61-93.

Traxler, J. (2009). Current State Of mobile learning. In ALLY, M. (Ed.), Mobile learning: Transforming the delivery of education and training. Edmonton: AU Press. 9-24.

Zurita, G., Nussbaum, M. (2004). Computer supported collaborative learning using wirelessly interconnected hand-held computers. Computers \& Education. 